\title{
X-ray Pulsar Navigation Algorithms and Testbed for SEXTANT ${ }^{1}$
}

\author{
Luke M. B. Winternitz ${ }^{2}$, Munther A. Hassouneh ${ }^{3}$, Jason W. Mitchell ${ }^{4}$, \\ Jennifer E. Valdez ${ }^{5}$, Samuel R. Price ${ }^{6}$, Sean R. Semper ${ }^{7}$, Wayne H. Yu ${ }^{8}$, \\ Paul S. Ray ${ }^{9}$, Kent S. Wood ${ }^{10}$, Zaven Arzoumanian ${ }^{11}$, Keith C. Gendreau ${ }^{12}$
}

\begin{abstract}
The Station Explorer for X-ray Timing and Navigation Technology (SEXTANT) is a NASA funded technologydemonstration. SEXTANT will, for the first time, demonstrate real-time, on-board X-ray Pulsar-based Navigation (XNAV), a significant milestone in the quest to establish a GPS-like navigation capability available throughout our Solar System and beyond.

This paper describes the basic design of the SEXTANT system with a focus on core models and algorithms, and the design and continued development of the GSFC X-ray Navigation Laboratory Testbed (GXLT) with its dynamic pulsar emulation capability. We also present early results from GXLT modeling of the combined NICER X-ray timing instrument hardware and SEXTANT flight software algorithms.
\end{abstract}

\section{Table of Contents}

\begin{tabular}{|c|c|}
\hline & INTRODUCTION... \\
\hline & X-Ray Pulsar Navigation .. \\
\hline & THE NICER X-RAY TIMing InSTRUMENT ..... \\
\hline & SEXTANT SYSTEM ARCHITECTURE OVERVIEW \\
\hline & SEXTANT CORE MODELS AND ALGORITHMS \\
\hline & GXLT END-TO-END SIMULATION ............ \\
\hline & GXLT HARDWARE-IN-THE-LOOP MODE....... \\
\hline & Preliminary Simulation ReSUlts .......... \\
\hline 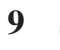 & SUMMARY AND FUTURE WORK........... \\
\hline $\mathbf{0}$ & ACKNOWLEDGMENT $\ldots . . . \ldots \ldots \ldots$ \\
\hline
\end{tabular}

\footnotetext{
${ }^{1}$ This is a work of the U.S. Government and is not subject to copyright protection in the United States.

${ }^{2}$ Sr. Navigation Engineer, NASA GSFC, Code 596, Greenbelt, MD 20771, USA, luke.b.winternitz@nasa.gov

${ }^{3}$ Flight Systems Engineer, NASA GSFC, Code 596, Greenbelt, MD 20771, USA, monther.a.hasouneh@nasa.gov

${ }^{4}$ Sr. Navigation Engineer, NASA GSFC, Code 595, Greenbelt, MD 20771, USA, jason.w.mitchellenasa.gov

${ }^{5}$ Aerospace Engineer, NASA GSFC, Code 596, Greenbelt, MD 20771, USA, jennifer.valdez@nasa.gov

${ }^{6}$ Software Engineer, NASA GSFC, Code 596, Greenbelt, MD 20771, USA, samuel.r.price@nasa.gov

${ }^{7}$ Aerospace Engineer, NASA GSFC, Code 596, Greenbelt, MD 20771, USA, sean.r.semper@nasa.gov

${ }^{8}$ Aerospace Engineer, NASA GSFC, Code 595, Greenbelt, MD 20771, USA, wayne.h.yu@nasa.gov

${ }^{9}$ Astrophysicist, Space Science Division, Naval Research Laboratory, Washington, DC 20375, USA, paul.rayenrl. navy . mil

${ }_{10}$ Astrophysicist, Space Science Division, Naval Research Laboratory, Washington, DC 20375, USA, kent . woodenrl.navy.mil

${ }^{11}$ NICER Deputy PI, CRESST/NASA GSFC/USRA/662, Greenbelt, MD 20771, zaven.arzoumanianenasa.gov

${ }^{12}$ NICER PI, NASA GSFC, Code 662, Greenbelt, MD 20771, USA, keith.c.gendreau@nasa.gov
}

\section{INTRODUCTION}

SEXTANT is a NASA Space Technology Mission Directorate Game Changing Development Program Office funded technology-demonstration enhancement to the Neutron-star Interior Composition Explorer (NICER) mission. NICER is a NASA Science Mission Directorate funded X-ray Astrophysics Explorer Mission of Opportunity that will be hosted on the International Space Station (ISS). SEXTANT will, for the first time, demonstrate real-time, on-board Xray Pulsar-based Navigation (XNAV), a significant milestone in the quest to establish a GPS-like navigation capability available throughout our Solar System and beyond. The SEXTANT demonstration will exploit the large collecting area $\left(>1800 \mathrm{~cm}^{2}\right)$, low background $(<0.2$ counts $/ \mathrm{s})$, and precise timing $(<100 \mathrm{~ns} 1-\sigma)$ provided by NICER's X-ray timing instrument.

SEXTANT will demonstrate the use of Millisecond Pulsars (MSPs), rapidly spinning neutron stars, as deep-space navigation beacons which could someday guide humankind out of Earth orbit, to destinations throughout the Solar System and beyond. SEXTANT's primary objective is to demonstrate real-time orbit determination with uncertainty better than $10 \mathrm{~km}$ with 2 weeks of measurements in the highly dynamic Low-Earth Orbit (LEO) of the ISS. SEXTANT also includes the development of a ground testbed, called the GXLT, that enables real-time and faster than real-time simulation of navigation scenarios. With the addition of a unique Modulated X-Ray Source (MXS), flight-like X-ray detector, and timetagging electronics, this testbed provides a test-as-you-fly hardware-in-the-loop simulation capability.

The SEXTANT demonstration is a technology enhancement to the NICER mission, which is an X-ray Astrophysics Mission of Opportunity to ISS that is currently in Phase C [1] and plans to launch in October 2016. NICER will undertake a fundamental investigation of extremes in gravity, material density, and electromagnetic fields of rapidly spinning neutron stars via time-resolved X-ray spectroscopy $[2,3]$. NICER achieves this objective by deploying an X-ray telescope instrument as an attached payload on a zenith-side ExPRESS Logistics Carrier (ELC) aboard the ISS, Figure 1. NICER offers over an order-of-magnitude improvement in time-coherent sensitivity and timing resolution beyond the capabilities of any X-ray observatory flown to date. As a flight software augmentation to NICER, SEXTANT will use a subset of the data collected for the NICER science program, on-board and in real-time, to accomplish its objective.

NICER and SEXTANT are funded through a cost-sharing opportunity between the NASA Science Mission Directorate and NASA Space Technology Mission Directorate Game Changing Development Program Office.

In the following, an introduction to X-ray pulsar navigation is given, followed by a brief description of the NICER X- 


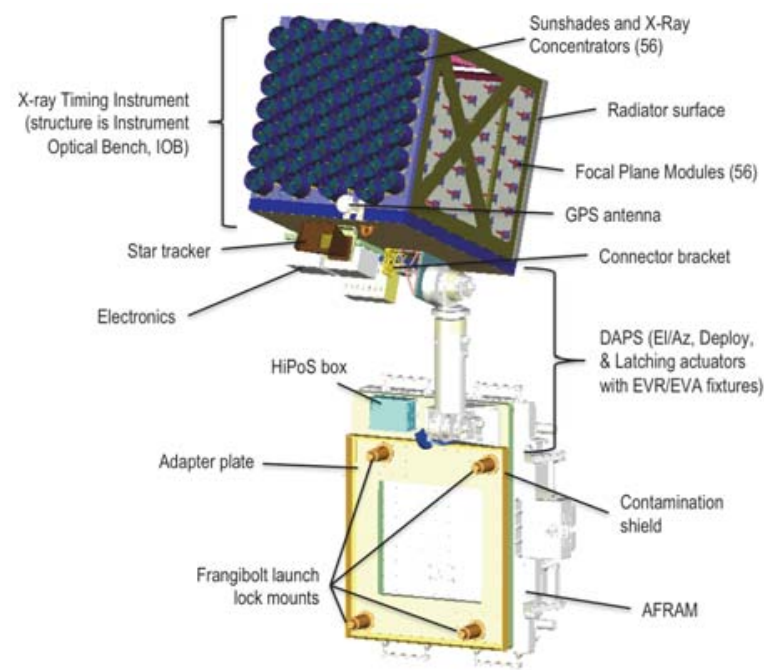

Figure 1: Neutron-star Interior Composition Explorer (NICER) payload reference representation.

ray timing instrument and the SEXTANT architecture. Then, details of the SEXTANT core models and algorithms, and end-to-end simulation are discussed. This is followed by a description of the GXLT hardware components and a brief presentation of prelimiary simulation results. Finally, the paper concludes with a summary of ongoing and future work.

\section{X-Ray Pulsar Navigation}

X-ray observations of celestial sources can provide useful navigation information to spacecraft in a range of applications from LEO to interplanetary, and even interstellar, space. One source of such information are X-ray emitting pulsars, which are neutron stars whose X-ray emission is modulated at the rotational period of the star. In this paper, we define the X-ray Pulsar Navigation (XNAV) concept as spacecraft navigation using X-ray observations of such pulsars. A subset of pulsars, the millisecond pulsars, are highly stable clocks, with long term stability comparable to laboratory atomic clocks. For these pulsars, a simple physical model with a small number of parameters can predict the arrival time of pulses to microsecond accuracy over months or years. A measurement of the difference between the arrival time of a pulse at a spacecraft and the predicted arrival time according to an onboard navigation solution can provide an error signal that can be used to measure the location of the spacecraft in a manner similar to GPS [4-6] (see Figure 2).

The use of radio pulsars as navigation beacons was first considered shortly after their discovery [7]. The idea was later extended to X-rays using the earliest established X-ray pulsars [8], but the achievable accuracy was severely limited by the noise characteristics of the X-ray pulsars known at the time. The first X-ray instrument with a specific goal of exploring X-ray navigation techniques was the Unconventional Stellar Aspect (USA) Experiment, flown in 1999 on the DoD ARGOS satellite, under the Space Test Program [9]. This experiment explored a broader concept of X-ray navigation, not limited to pulsars and also not limited to position determination but considering also time transfer and aspect determination.
During the 1990s X-rays began to be detected from millisecond pulsars previously known only as radio pulsars [10]. This development greatly improved the expected accuracy of an XNAV system and spurred detailed studies [4], resulting in a patent on the idea (US Patent 7,197,381). A DARPA program emphasizing millisecond pulsar methodologies represented the next stage of DoD development, and it was during this program that the first laboratory facility to simulate X-ray pulsars was developed at GSFC. By now the concept of Xray navigation is being pursued in several other countries as well as the U.S $[11,12]$.

XNAV has the potential to become an enabling technology for very deep space exploration missions and an important augmentation to NASA's Deep Space Network (DSN), the current standard for interplanetary navigation and communication [13].

\section{THE NICER X-RAY TIMING INSTRUMENT}

The key measurable for an XNAV instrument are pulse arrival times determined from a set of detected X-ray photons. The instrument must be designed to be able to produce a high signal-to-noise pulse profile in an integration time that is short compared to the timescale for a propagated orbit to deviate from the true trajectory by more than the required navigation accuracy. In turn, this requires a detector with the following characteristics: large effective area in the region of the X-ray spectrum where MSP pulsations can be observed (roughly $0.2-8 \mathrm{keV}$ ), high precision time tagging of each $\mathrm{X}$-ray photon, and low background rates. To be useful in future spacecraft navigation applications, this should be achieved with the lowest possible mass, volume, and power requirements.

The NICER X-ray Timing Instrument (XTI) (Figure 3) is extremely well suited to this task. It is a modular array of 56 identical telescopes, making it easily scalable to a range of potential applications. Each telescope consists of a lightweight grazing-incidence optic made up of concentric foil mirrors. The mirrors concentrate $\mathrm{X}$-rays onto a small (1 mm radius unobstructed circular aperture) detector area using a single bounce, in contrast to typical (e.g. Wolter I; [14]) imaging X-ray optics that require two bounces and thus incur a significant efficiency penalty to achieve quality imaging. The XTI provides over $1800 \mathrm{~cm}^{2}$ of area in a package with frontal area of $6400 \mathrm{~cm}^{2}$, an areal efficiency of $28 \%$.

The X-ray detectors are commercial (Amptek) silicon drift detectors that have very high quantum efficiency over the photon energy range of interest. The detectors are read out by dual-channel electronics chains that provide both high time resolution (100 ns) and excellent spectral resolution (120 $\mathrm{eV}$ ) with very low dead time. Particle backgrounds are low because of the small detector volume made possible by the concentrating optics, while particles that do interact in the detector can be rejected at high efficiency by their energy deposition and by filtering events that occurred outside of the illuminated area of the detector by a comparing the pulse heights determined by the slow and fast electronics channels. In addition to reducing particle backgrounds, the focusing of the mirrors reduces the backgrounds from the cosmic diffuse X-ray background and neighboring sources that are outside the $\sim 6$ arcmin (FWHM) field of view. The expected background rate in the critical $0.4-2 \mathrm{keV}$ band is $<0.2$ counts per second. 

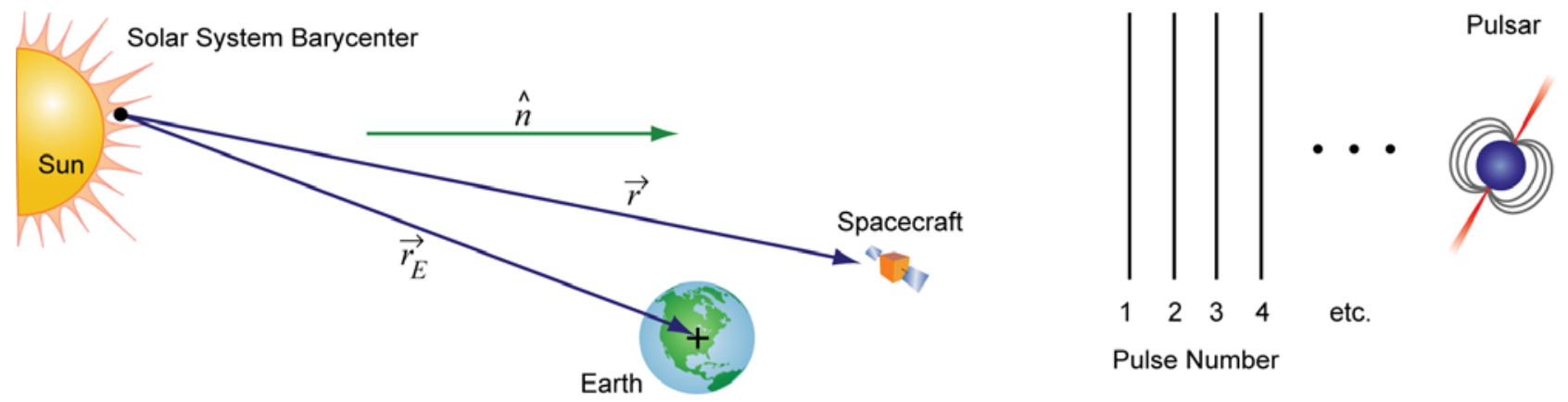

Figure 2: Geometry of an XNAV observation.

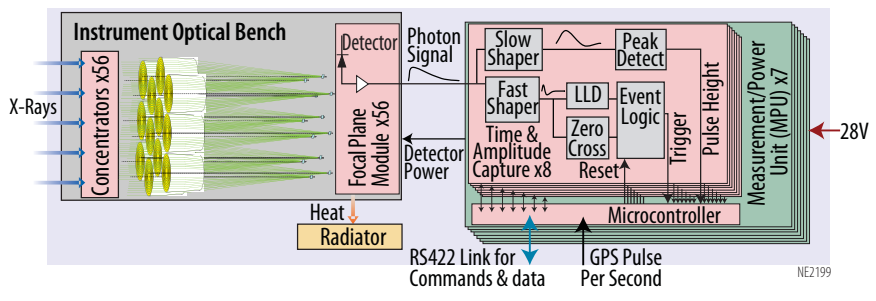

Figure 3: Block diagram of NICER XTI showing the main components: concentrators, focal plane modules, detectors and measurement and power unit.

\section{SEXTANT SySTEM ARCHITECTURE OVERVIEW}

The main components of the SEXTANT system and the data flow relationship among these components are shown in Figure 4. A detailed overview of the SEXTANT architecture can be found in [15]. The components that are external to the NICER XTI are briefly summarized below.

\section{SEXTANT flight software}

The X-ray Pulsar Navigation Flight Software (XFSW) is a single application hosted by the NICER Instrument Flight Software (IFSW). It pre-processes and buffers photon events from the XTI until a sufficient number of events from a single pulsar is collected. The photon events are then batch processed using a Maximum Likelihood (ML) estimation algorithm to produce a single pulse phase and Doppler measurement. The Goddard Enhanced Onboard Navigation System (GEONS) navigation filter [16], seeded by a single (intentionally degraded) initial state estimate from the NICER GPS receiver, blends models of spacecraft dynamics with these pulse phase and Doppler measurements to maintain an estimate of the relevant spacecraft state, i.e., position, velocity, drag coefficient, etc.

\section{SEXTANT ground system}

The SEXTANT ground system maintains the pulsar catalog that provides current timing models, or ephemerides, and pulse profile templates as needed for navigation processing. The ground system is also responsible for performance monitoring and telemetry collection for post-processing purposes.

The pulsar catalog consists of a list of SEXTANT pulsars, with their associated timing models and X-ray lightcurve templates. The current baseline SEXTANT pulsar list is provided in Table 1, along with some basic parameters. Templates for six of the catalog pulsars, as constructed from archival Xray data retrieved from NASA's High Energy Astrophysics Science Archive Research Center (HEASARC) [17], are shown in Figure 5. The ground system will measure the phase relationship between the radio and X-ray templates for each pulsar, and track variations in the pulsar dispersion measure that are needed to maintain this alignment.

The timing models will be regularly updated using Time-ofArrival (TOA) data from external observatories, and combined with XTI data once NICER is operational. The ground system relies on the TEMPO2 pulsar timing software [18, 19] for the generation of timing models by fitting parameterized models to measured radio and X-ray pulse TOAs, and for generating the polynomial fits to the full models that are used for onboard processing.

\section{SEXTANT ground testbed}

The GXLT is a unique software and Hardware-in-the-Loop (HWIL) environment for development and testing of various XNAV algorithms and detector technologies. This allows the main SEXTANT core models and algorithms, as described in Section 5, to be exercised in a flight-like environment without a full-scale NICER XTI. The ground testbed is described in detail in Sections 7 and 8.

\section{SEXTANT CORE MOdelS AND Algorithms}

\section{Photon arrival model}

The fundamental data provided to the SEXTANT XNAV algorithms are the observed photon event timestamps (in GPS time, accurate to $100 \mathrm{~ns}$ RMS) with associated pulse heights, proportional to photon energy. In this section, we present the formal statistical model used to model this process.

The observed photon event timestamps $\left\{T_{k}\right\}_{k=1}^{N}$ received on-board NICER from the XTI are modeled as the arrival times of a Non-Homogeneous Poisson process (NHPP) with time varying mean cumulative count function $\bar{\Lambda}(t)[20,21]$. For such process, the probability of $k$ events arriving in the interval $\left(t_{a}, t_{b}\right)$ is given by

$$
P\left(k ;\left(t_{a}, t_{b}\right)\right)=\frac{e^{-\left(\bar{\Lambda}\left(t_{b}\right)-\bar{\Lambda}\left(t_{a}\right)\right)}\left(\bar{\Lambda}\left(t_{b}\right)-\bar{\Lambda}\left(t_{a}\right)\right)^{k}}{k !} .
$$

We assume the existence of a "rate function" $\bar{\lambda}$ such that $\bar{\Lambda}(t)=\int_{0}^{t} \bar{\lambda}(s) d s$. We further assume that the rate function 


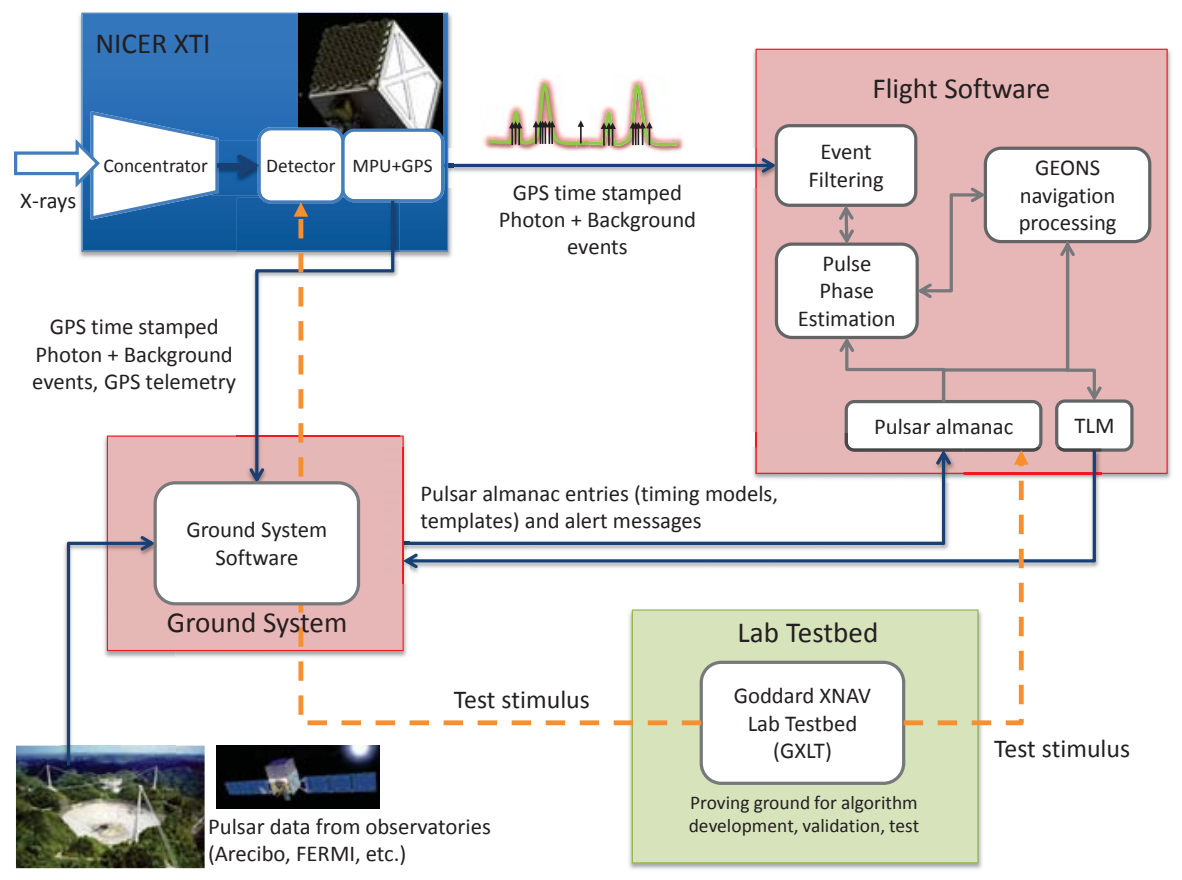

Figure 4: SEXTANT system architecture.

Table 1: SEXTANT pulsar catalog with basic parameters

\begin{tabular}{|c|c|c|c|c|c|c|}
\hline Name & $\begin{array}{l}\text { Period }(P) \\
\quad(\mathrm{ms})\end{array}$ & $\begin{array}{c}D^{a} \\
(\mathrm{kpc})\end{array}$ & $\begin{array}{c}\text { Source Pulsed Rate } \\
(\alpha, \mathrm{cnts} / \mathrm{s})\end{array}$ & $\begin{array}{c}\text { Total Bkg Rate } \\
(\beta, \text { cnts } / \mathrm{s})\end{array}$ & $\begin{array}{l}I_{p} \\
(-)\end{array}$ & $\begin{array}{c}\sigma^{c} \\
(\mu s)\end{array}$ \\
\hline Crab Pulsar & 33.51 & 2.0 & 660.000 & 13860.20 & 56963.3 & $6.6(10)^{b}$ \\
\hline B $1937+21$ & 1.56 & 5.0 & 0.029 & 0.24 & 27.0 & 14.1 \\
\hline B1821-24 & 3.05 & 4.9 & 0.093 & 0.22 & 231.2 & 9.5 \\
\hline $\mathrm{J} 0218+4232$ & 2.32 & 5.8 & 0.082 & 0.20 & 5.7 & 45.8 \\
\hline $\mathrm{J} 0030+0451$ & 4.87 & 0.3 & 0.193 & 0.20 & 5.4 & 98.8 \\
\hline $\mathrm{J} 1012+5307$ & 5.26 & 0.7 & 0.046 & 0.20 & - & - \\
\hline J0437-4715 & 5.76 & 0.2 & 0.283 & 0.62 & 2.9 & 159.4 \\
\hline J2124-3358 & 4.93 & 0.3 & 0.074 & 0.20 & - & - \\
\hline $\mathrm{J} 2214+3000$ & 3.12 & 1.5 & 0.029 & 0.26 & - & - \\
\hline J0751+1807 & 3.48 & 0.4 & 0.025 & 0.22 & - & - \\
\hline J1024-0719 & 5.16 & 0.5 & 0.015 & 0.20 & - & - \\
\hline
\end{tabular}

has the form

$$
\bar{\lambda}(t)=\lambda(\phi(t))=\beta+\alpha h(\phi(t))
$$

with $\alpha \geq 0, \beta \geq 0$ constant, and $h$ a nonnegative, continuous and piecewise differentiable real function, periodic with period $1(h(\phi+m)=h(\phi)$ for all integers $m)$ and with unit area $\int_{0}^{1} h(\phi) d \phi=1$ (see Fig. 5 for six pulsar profiles from the SEXTANT catalog). Finally, $\phi$ is assumed smooth with bounded positive derivative, bounded away from zero, so that $\phi^{-1}$ exists and is smooth.

The parameters $\alpha, \beta$ represent the average signal and total background count rates in units of counts per second, respectively. The function $h$ describes the characteristic shape of the pulsar intensity profile or lightcurve, see Figure 5. Finally, $\phi$ represents the evolution of the phase of pulsation with respect to the time parameter $t$ as seen at the detector.

\section{Pulse arrival model}

The phase at the detector $\phi$ is modeled as

$$
\phi(t)=\phi_{0}(t-\tau(t)),
$$

where $\phi_{0}$ is the phase evolution at a hypothetical reference observatory and $\tau(t)$ is the light propagation time of the pulse wavefront moving from the detector to the reference observatory. SEXTANT's flight software and end-to-end simulation currently uses either a Geocenter or Solar System Barycenter (SSB) reference observatory. In the former case, $c \tau(t) \simeq$ $\vec{n} \cdot \vec{x}(t)$, where $\vec{x}(t)$ gives the detector coordinates in a frame centered at the reference observatory and $\vec{n}$ is the direction to the given pulsar, to a sufficiently accurate approximation. In the latter case, parallax and Solar Shapiro delay terms are 


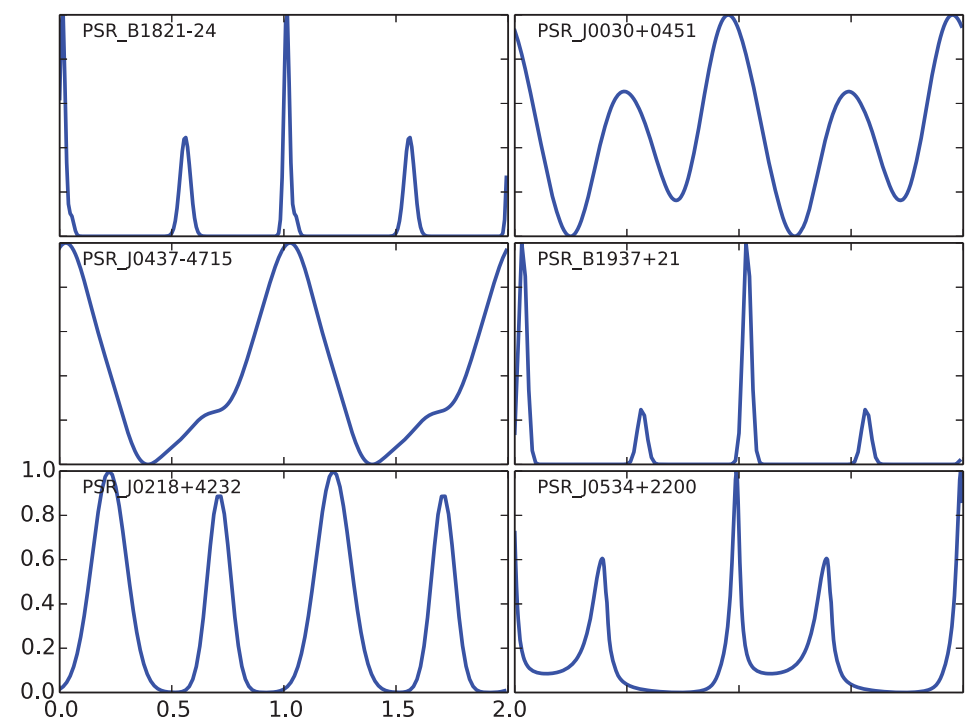

Figure 5: Current set of template profiles for XNAV pulsars. Two cycles of each pulsar are shown.

additionally needed to achieve similar accuracy.

SEXTANT relies on the pulsar timing software TEMPO2 [18] to provide a model for phase evolution at a reference observatory. TEMPO2 models are physically motivated leastsquares fits to radio observatory data of the form

$$
\phi_{0}(t)=p(t-\Delta(t)),
$$

with $p$ a quadratic or cubic polynomial shifted by timing correction $\Delta(t)$ which includes terms for Roemer, Einstein, and Shapiro, and binary delays. ${ }^{2}$ TEMPO 2 also provides convenient piecewise polynomial approximations to the full timing model $\phi_{0}$, which are used by the SEXTANT flight software.

\section{Count rate models}

The pulsed count rate $\alpha$ observed from each pulsar with the NICER XTI is determined by the spectrum of the source and the response matrix of the instrument. These values will be determined with high accuracy during pre-launch and on-orbit calibration for each target in the SEXTANT pulsar catalog (Table 1).

The background count rate is composed of any unpulsed flux from the pulsar, other point sources in the field of view, diffuse X-ray flux in the aperture, and unrejected radiation background. The latter component is expected to vary with position and time, and is the sum of the particle (proton, electron, positron) interactions and those originating with high-energy $(>30 \mathrm{keV}) \mathrm{X}$-rays and $\gamma$-rays that penetrate the instrument enclosure and produce secondary radiation within the XTI. The current SEXTANT simulations assume a constant background rate over each observation interval, but work is underway to remove this simplification and update $\beta$ to account for on-orbit particle background variability.

${ }^{2}$ The latter term accounts for the orbital motion and relativistic effects for pulsars in binary systems, which constitutes the majority of pulsars in the SEXTANT catalog. This effect prevents (3) from being well approximated by a linear function in time over typical observation timescales.

\section{Pulse phase and Doppler estimation}

The baseline SEXTANT algorithms work by observing several MSPs from the SEXTANT catalog cyclically and when sufficient time is accumulated on a particular MSP, the collected photon events are batch processed to extract pulse phase and Doppler estimates, which are finally passed to a navigation filter.

We would like to use standard parameter estimation techniques to generate the phase and Doppler estimates. In general, due to the spacecraft motion and the form of $\phi_{0}$ (especially with the binary terms), the phase evolution has a complicated form that is difficult to directly parameterize with a low-order model. For example, a periodic NHPP model, used in some prior work, would be inadequate in most cases. To address this, as described in the following, our approach assumes the existence of an approximation to the phase evolution that captures most of the dynamics and we then parameterize a correction to this approximation.

Specifically, we assume existence of a smooth approximation $\tilde{\phi}$ such that $\phi(t)=\tilde{\phi}(t)+e(t)$, so that $\left\{T_{k}\right\}$ is NHPP with rate $\lambda(\tilde{\phi}(t)+e(t))$. Furthermore we assume over the observation interval $\left[t_{a}, t_{b}\right], e(t)$ can be fit by a low-order parametric model.

\section{The phase prediction $\tilde{\phi}$}

Using a predicted position $\tilde{\vec{x}}(t)$, provided by the GEONS navigation filter, we expand the phase function as

$$
\begin{aligned}
\phi(t) & =\phi_{0}\left(t-\frac{\vec{x}(t) \cdot \vec{n}}{c}\right) \\
& =\phi_{0}\left(t-\frac{\tilde{\vec{x}}(t) \cdot \vec{n}}{c}+\frac{\delta \vec{x}(t) \cdot \vec{n}}{c}\right) \\
& \simeq \tilde{\phi}(t)+\dot{\phi}_{0}\left(t-\frac{\tilde{\vec{x}}(t) \cdot \vec{n}}{c}\right) \frac{\delta \vec{x}(t) \cdot \vec{n}}{c}, \\
& =\tilde{\phi}(t)+e(t),
\end{aligned}
$$


where $\vec{x}$ is the spacecraft position vector, $\vec{n}$ is the unit vector direction to the pulsar, $c$ is the speed of light, $\delta \vec{x}(t)=\tilde{\vec{x}}(t)-$ $\vec{x}(t)$, and $e(t):=\dot{\phi}_{0}\left(t-\frac{\tilde{\vec{x}}(t) \cdot \vec{n}}{c}\right) \frac{\delta \vec{x}(t) \cdot \vec{n}}{c}$; see observation geometry shown in Figure 2. We assume $e(t)$ is well fit by a linear model, i.e., $e(t) \simeq q+f\left(t-t_{a}\right)$ for constant $q$ and $f^{3}$

In summary, the SEXTANT phase and Doppler estimation routine proceeds as follows.

1. Observe arrival times $\left\{t_{k}\right\}_{k=1}^{N}$ during a fixed interval $\left[t_{a}, t_{b}\right]$.

2. Determine estimates $(\hat{q}, \hat{f})$ of the parameters $(q, f)$ in the model $e(t)=q+f\left(t-t_{a}\right)$.

3. Form phase and Doppler estimates $\hat{\phi}(t)=\tilde{\phi}(t)+\hat{q}+$ $\hat{f}\left(t-t_{a}\right)$ and $\hat{\dot{\phi}}(t)=\hat{f}$, respectively.

Step 2 is clarified below.

\section{Pulse phase estimation routines}

As described previously, the photon time-of-arrival process $\left\{T_{k}\right\}_{k=1}^{N}$ observed during the interval $\left[t_{a}, t_{b}\right]$ is assumed to follow a NHPP with rate

$$
\bar{\lambda}(t ; q, f):=\lambda\left(\tilde{\phi}(t)+q+f\left(t-t_{a}\right)\right),
$$

and our goal is to estimate the parameters $(q, f)$. Toward this end, it can be shown that the arrival phases admit the joint probability density function

$$
p\left(t_{1}, t_{2}, \ldots t_{N} ; q, f\right)=e^{-\int_{t_{a}}^{t_{b}} \bar{\lambda}(s) d s} \Pi_{k=1}^{N} \bar{\lambda}\left(t_{k}\right)
$$

with the constraint $t_{a}<t_{1}<t_{2} \ldots<t_{N}<t_{b}$, where $N$ is also a random variable here (see e.g., [6, 23, 24]).

An approximate $M L E$-The exponent in (7) is the mean total count rate, which approximately equals $(\alpha+\beta)\left(t_{b}-t_{a}\right)$, and is thus considered independent of the parameters $(q, f)$, as long as the observation interval includes many cycles of pulsation. This is always the case for SEXTANT MSP observations, therefore, this term may be dropped leading to the approximate MLE

$$
(\hat{q}, \hat{f})=\arg \max _{q, f} \sum_{k=1}^{N} \log \lambda\left(\tilde{\phi}\left(T_{k}\right)+q+f\left(T_{k}-t_{a}\right)\right) .
$$

\section{Measurement uncertainty}

Here we discuss the components of the error in the phase and Doppler estimates. An overall estimate of the level of measurement noise is a required input parameter for the navigation filter. For SEXTANT we specify a measurement noise $(1 \sigma)$ level corresponding to two times the square-root of Cramér-Rao Lower Bound (CRLB), described next.

Poisson noise-Given sufficient observation time, the ML described above will achieve the CRLB for model (7) with

\footnotetext{
${ }^{3}$ As suggested in [22], an approximation better matched to the orbital dynamics would be $\delta \vec{x}(t)=\Phi\left(t, t_{a}\right) \delta \vec{x}\left(t_{a}\right)$, where $\Phi\left(t, t_{a}\right)$ is the state transition matrix provided by the navigation filter. In this case, we would estimate a 6-dimensional parameter vector $\delta \vec{x}\left(t_{a}\right)$. We leave this for future investigation because the linear model has been shown to work well in simulation.
}

parameters $\theta=(q, f)$ (e.g., [25]). It is shown in [6, 24] to be given by

$$
\operatorname{CRLB}(\theta)=\frac{2}{I_{p}}\left[\begin{array}{cc}
\frac{2}{T} & \frac{-3}{T^{2}} \\
\frac{-3}{T^{2}} & \frac{6}{T^{3}}
\end{array}\right],
$$

with $T=t_{b}-t_{a}$, and where constant $I_{p}$ is

$$
I_{p}:=\int_{0}^{1} \frac{(\alpha \dot{h}(\phi))^{2}}{\beta+\alpha h(\phi)} d \phi .
$$

For SEXTANT, due to a constraint imposed by the navigation filter, we always refer the measurement to the time at end of the observation interval ${ }^{4}$ and get

$$
\hat{\phi}\left(t_{b}\right)=\tilde{\phi}\left(t_{b}\right)+\hat{q}+\hat{f}\left(t_{b}-t_{a}\right) .
$$

Subtracting this equation from $\phi\left(t_{b}\right)$ and using the assumed form of the prediction error we get that the phase and frequency estimate errors are $q-\hat{q}+(f-\hat{f}) T$ and $(f-\hat{f})$, respectively. Thus using (9), we get $\frac{4}{I_{p} T}$ and $\frac{12}{I_{p} T^{3}}$, respectively, for the phase and frequency error variances.

Beyond Poisson statistics and the CRLB, there are additional sources of error in the derived state vector estimates that should be considered. Addition of the following terms are being developed as enhancements to the current baseline SEXTANT models and simulations. The first three amount to errors in the pulsar almanac parameters $\phi_{0}, \alpha, \beta$, and $h$. We strive to control these error sources to be smaller than the Poisson noise contribution, which partially justifies the use of twice the CRLB for the filter measurement noise.

Timing noise and glitches-Pulsar timing noise and glitches (abrupt shifts in pulse frequency) are both unpredictable processes that limit the accuracy of extrapolated timing models. These effects are most pronounced for young pulsars (such as the Crab pulsar), but even the very stable millisecond pulsars usually have measurable timing noise and occasionally even suffer small glitches (as seen in the XNAV pulsar B1821-24). For glitches, the only mitigation is to monitor the timing of each of the pulsars and stop using it for navigation when a glitch is detected, only returning it to operation when a sufficiently stable post-glitch solution is determined. For timing noise, the effects can be minimized by updating the timing models frequently based on recent observations (to limit the time the pulsar has to wander away from the current solution), and to use optimal techniques for extrapolating pulsar phase predictions in the presence of timing noise, as described in [26].

Uncertainty in radio to $X$-ray offset-Initially, the timing models for XNAV pulsars will be developed from groundbased radio observations, but the phase measurements are made in the X-ray band. Therefore, the absolute phase offset between the X-ray and radio pulse profiles must be known precisely. This offset can be measured, through contemporaneous measurements with $\mathrm{X}$-ray and radio telescopes, to an accuracy limited by signal-to-noise of each measurement and the cross calibration of the reference clocks used by each observatory. Additionally, the radio pulse arrival times must be corrected for the effects of dispersion. This can be done with careful measurements at multiple radio frequencies. However, any variations from the measured value introduce an error in the phase predictions at infinite frequency (e.g.

\footnotetext{
${ }^{4}$ Although, referring it to the middle of the interval would improve the
} estimate. 
X-ray). These variations can come from several sources including the interstellar medium (as the pulsar moves through the Galaxy at high velocity), the interplanetary medium, the solar wind, and the Earth's ionosphere.

Astrometric errors-The measured astrometric parameters for the pulsars (position, proper motion, and parallax) are used to compute the time offset between the spacecraft and the reference observatory. These quantities are measured via timing (or interferometric) observations at $1 \mathrm{AU}$ from the Sun (i.e. on Earth). As the spacecraft moves to much larger distances from the Sun, the accuracy required on these parameters increases but this is not a concern to SEXTANT.

Non-timing model mismatch-Errors in the pulse template mismatch and source and background flux rates (if they are not estimated, which they will be in the final SEXTANT algorithms) will introduce additional error in the estimation process.

Non-ideal behavior of the reference clock-The events provided by the NICER XTI are timestamped to GPS time to $100 \mathrm{~ns}$ accuracy. The SEXTANT baseline implementation uses the full accuracy of these timestamps, effectively providing continuous calibration of its onboard clock. An XNAV sensor operating outside of the range of GPS will timestamp event times with respect to an on-board clock which will drift over time. This error can be compensated either by periodically recalibrating via contact with the ground system or by enhancing the measurement model to allow estimation of clock states, or a combination of both. While our baseline approach is idealized, it is sufficiently representative for the purposes of the demonstration. Moreover, non-ideal reference clock behavior will be investigated on the ground by intentionally degrading stored science telemetry.

\section{Event simulation}

The ability to efficiently simulate NHPP photon events is a core function of the GXLT necessary for developing and testing the SEXTANT flight software algorithms.

An important property of Poisson processes, relevant to simulation, is that given a standard (with constant rate 1) Poisson process $\left\{S_{k}\right\}$, the process $T_{k}=\Lambda^{-1}\left(S_{k}\right)$ is an NHPP with mean cumulative count function $\Lambda(t)$.

Thus, an NHPP with rate $\bar{\lambda}(t)=\lambda(\phi(t))$ can be generated by $T_{k}=\bar{\Lambda}^{-1}\left(S_{k}\right)$ where $\left\{S_{k}\right\}$ is a standard Poisson process (with constant rate 1 ) and

$$
\bar{\Lambda}(t)=\int_{0}^{t} \lambda(\phi(z)) d z .
$$

However, a direct inversion of $\bar{\Lambda}$, through table lookup, for example, would be computationally expensive and does not take advantage of the periodicity of $\lambda$. Considering a change of variable $u=\phi(z)$ in (12), we have

$$
\begin{aligned}
\bar{\Lambda}(t) & =\int_{\phi(0)}^{\phi(t)} \frac{\lambda(u)}{\dot{\phi}\left(\phi^{-1}(u)\right)} d u \\
& \simeq \frac{1}{\dot{\phi}\left(t_{m}\right)} \Lambda(\phi(t))=: \Lambda_{1}(\phi(t))
\end{aligned}
$$

where $\Lambda(t)=\int_{\phi(0)}^{\phi(t)} \lambda(u) d u$ and we assume without loss of generality that $\phi(0)=0$. We also assume $\dot{\phi}(t) \simeq \dot{\phi}\left(t_{m}\right)$ holds approximately over the simulation interval where $t_{m}$ is the midpoint of the observation interval, and we define $\Lambda_{1}:=$ $\Lambda / \dot{\phi}\left(t_{m}\right)$.

Now we can efficiently simulate $\left\{T_{k}\right\}$ by inverting $\bar{\Lambda}$ in two steps: first computing $U_{k}=\Lambda_{1}^{-1}\left(S_{k}\right)$, taking advantage of the periodicity of $\lambda_{1}=\dot{\Lambda}_{1}$, and then computing $T_{k}=$ $\phi^{-1}\left(U_{k}\right)$.

To take advantage of $\lambda_{1}$ 's periodicity, note that if $s=\Lambda_{1}(u)$ and $u=n+r$, with $n$ an integer and $r \in[0,1)$, then $s=n \Lambda_{1}(1)+\Lambda_{1}(r)$ so that $n=\left\lfloor\frac{s}{\Lambda_{1}(1)}\right\rfloor$, and $r=\Lambda_{1}^{-1}(s$ $\left.\bmod \Lambda_{1}(1)\right)$. Finally, $\Lambda_{1}^{-1}$ can be efficiently evaluated in $\left[0, \Lambda_{1}(1)\right)$ by interpolation of a lookup table.

The input sequence $\left\{S_{k}\right\}$ can be efficiently generated by summing Independent and Identically Distributed (IID) unitparameter exponential random variables $z_{k}$, which, in turn, may be generated simply as $z_{k}=-\log \left(1-v_{k}\right)$ with $v_{k} \stackrel{\text { iid }}{\sim}$ Uniform[0,1), (e.g., [25].)

In summary, this gives the following three step approach to event simulation used in the SEXTANT ground testbed and end-to-end simulation:

1. Simulate $N$ standard Poisson process event times $\left\{S_{k}\right\}_{k=1}^{N}$,

2. Transform these events to phase events $U_{k}=\Lambda_{1}^{-1}\left(S_{k}\right)$, 3. Finally transform to $T_{k}=\phi^{-1}\left(U_{k}\right)$.

The photon count increment $N$ should be chosen so that the approximation $\dot{\phi}(t) \simeq \dot{\phi}\left(t_{m}\right)$ is valid over the interval spanned by the event times $\left\{T_{k}\right\}_{k=1}^{N}$. If the simulation of a continuous observation of a single pulsar needs to be broken up to achieve this error condition, the interval breakpoints should coincide with a particular event time $T_{k}$, to ensure the process remains a valid NHPP.

There are other approaches to simulating NHPPs (see e.g.,[20]). In particular "accept/reject" methods are simple and quite general and may be preferred in some circumstances (time varying particle background rates), but are not expected to achieve the efficiency of the method described above.

\section{Filter measurement model}

In the SEXTANT navigation filter, the phase and Doppler measurements are blended with models of the spacecraft dynamics to update an estimate of the spacecraft state.

In order to do this, a measurement equation, describing how the spacecraft position $\vec{x}$ and velocity $\dot{\vec{x}}$ is related to the measurements is needed. For the phase measurement, assuming a Geocenter reference observatory (where parallax and Solar Shapiro delay are negligible), this is just the phase evolution equation

$$
\phi(t)=\phi_{0}(t-\vec{n} \cdot \vec{x}(t) / c)
$$

previously described in the current section.

The frequency measurement equation is obtained by differen- 
tiating (14), leading to

$$
\dot{\phi}(t)=\dot{\phi}_{0}(t-\Delta(t))\left[1-\frac{\vec{n} \cdot \dot{\vec{x}}(t)}{c}\right],
$$

with $\Delta(t):=\vec{n} \cdot \vec{x}(t) / c$.

The filter also requires the first order partial derivatives which are given by

$$
\frac{\partial \vec{y}}{\partial \vec{z}}=-\frac{1}{c}\left(\begin{array}{cc}
\dot{\phi}_{0}(t-\Delta(t)) \vec{n}^{\mathrm{T}} & 0 \\
(1-\dot{\Delta}(t)) \ddot{\phi}_{0}(t-\Delta(t)) \vec{n}^{\mathrm{T}} & \dot{\phi}_{0}(t-\Delta(t)) \vec{n}^{\mathrm{T}}
\end{array}\right),
$$

with $\vec{y}=(\phi, \dot{\phi})^{\mathrm{T}}$ and $\vec{z}=(\vec{x}, \dot{\vec{x}})$.

\section{GXLT END-TO-END Simulation}

The NASA GXLT, developed to support the SEXTANT demonstration, is a unique hardware and software test environment that allows for rapid, high-fidelity, end-to-end simulation and performance for arbitrary mission concepts and evaluation of various spacecraft XNAV scenarios. The GXLT leverages several Goddard Space Flight Center (GSFC) Guidance, Navigation, and Control (GN\&C) software tools and $\mathrm{X}$-ray detector lab technologies. The overall end-to-end simulation architecture of the SEXTANT ground testbed [27] is depicted in Figure 6, with the available simulation process flows per level indicated by the colored arrows.

\section{GXLT Simulation Flow}

The GXLT offers three levels of simulation, each with unique benefits, where the simulation process flows differ primarily in the way measurements are produced. These levels are enumerated and described below.

Level $0:$ navigation processing only-In this simulation mode, the phase and Doppler measurements are directly simulated by evaluating the measurement model and adding simulated noise. The photon arrival process is not simulated. As a standard approach for navigation performance studies, this approach is useful for long simulation studies, e.g., deep space trajectories, and for tuning navigation filter parameters. This flow is indicated by the red arrows in the upper path of Figure 6.

Level 1: software simulated photon arrival process-In this mode, the fidelity of measurement generation is increased by simulating the photon events in software. The photon arrival process is simulated to generate the photon events, which are then processed using Eq. (8). This flow is the primary development mode for SEXTANT, and is indicated by the green arrows in lower path of Figure 6.

Level 2: real-time, hardware-in-the-loop mode-In this mode, which is the subject of Section 7, the MXS is driven in real-time to produce X-rays, in the correct energy band, with arrival-time statistics that precisely follow the simulation truth profile. This dynamically modulated output of the MXS stimulates a customized Commercial Off-The-Shelf (COTS) detector package, a NICER Engineering Test Unit (ETU) flight detector, and eventually NICER flight detector(s).

Importantly, the modulation of the MXS incorporates the orbital dynamics of a simulated spacecraft, so that photon events from a static detector emulate a detector following the prescribed orbit trajectory Importantly, the MXS is specifically modulated so that photon events from a static detector appear to originate from a detector following a prescribed orbit trajectory.

The COTS detector package used by the GXLT hardware suite includes a commercial Silicon Drift Detector (SDD) from Amptek, Inc. and custom timing/readout electronics, which log timestamped photon events to a file. These events can then be processed by the flight software, and have previously been verified to match the statistics of those simulated in Level 1 [27]. This flow is indicated by the blue arrows along the center-line of Figure 6.

\section{Simulation procedure}

The simulation process for the flow of each level is described in the following.

Scenario definition-A simulation starts with the specification of a scenario configuration that defines the simulation level and length, X-ray optics/detector models, pulsar target list and models, target observation times, a truth ephemeris file, parameters for the orbit propagator, scheduling options and visibility constraints, event simulation options, photon processing algorithms, and finally, navigation filter options.

Truth Trajectory generation- Next, a truth spacecraft ephemeris is read or generated based on the scenario options. The SEXTANT end-to-end simulation can use an external ephemeris file generated by an arbitrary source, or alternately run the NASA open source General Mission Analysis Tool (GMAT) software to propagate a trajectory on the fly. The force models includes a 40x40 EGM96 earth gravity field, third-body perturbations for the Sun and Moon, and the MSISE90 atmospheric drag model. ISS trajectory data is made available to the public by the NASA Johnson Space Center (JSC)'s Flight Design and Dynamics Division [28]. The available ISS real-time data include: mass (weight), area, drag, monthly solar flux and geomagnetic index, maneuver information for the current ephemeris, state vectors, two-line mean element sets, etc.

Visibility and Scheduling-Next, the visibility of target pulsars is calculated at each simulation timestep. This process considers occultations by the Sun, Earth, and Moon; instrument constraints that limit achievable azimuth and elevation angles; and particle background limits within the South Atlantic Anomaly (SAA).

Then, based on visibility, a pulsar observation schedule is generated that attempts to reduce the state residuals by balancing pulsar target priorities within pulsar viewing periods. Significant observation times per pulsar navigation measurement, due to signal noise require that observations of each pulsar, are grouped to avoid stagnant navigation data. At the same time, each pulsar navigation measurement must also be timed to arrive when needed to reduce state residuals. Instrument hardware slew rate limits are also included in the schedule.

Truth observable generation-At each time step, the detector pulse phase model is evaluated using the model at the reference observatory and the spacecraft ephemeris. For a geocentric reference observatory, we simply evaluate the phase model, as referenced to Universal Coordinated Time (UTC), at the reference observatory and account for the Roemer, or geometric, delay. For an SSB reference observatory, we transform the spacecraft state to barycentric coordinates and time 


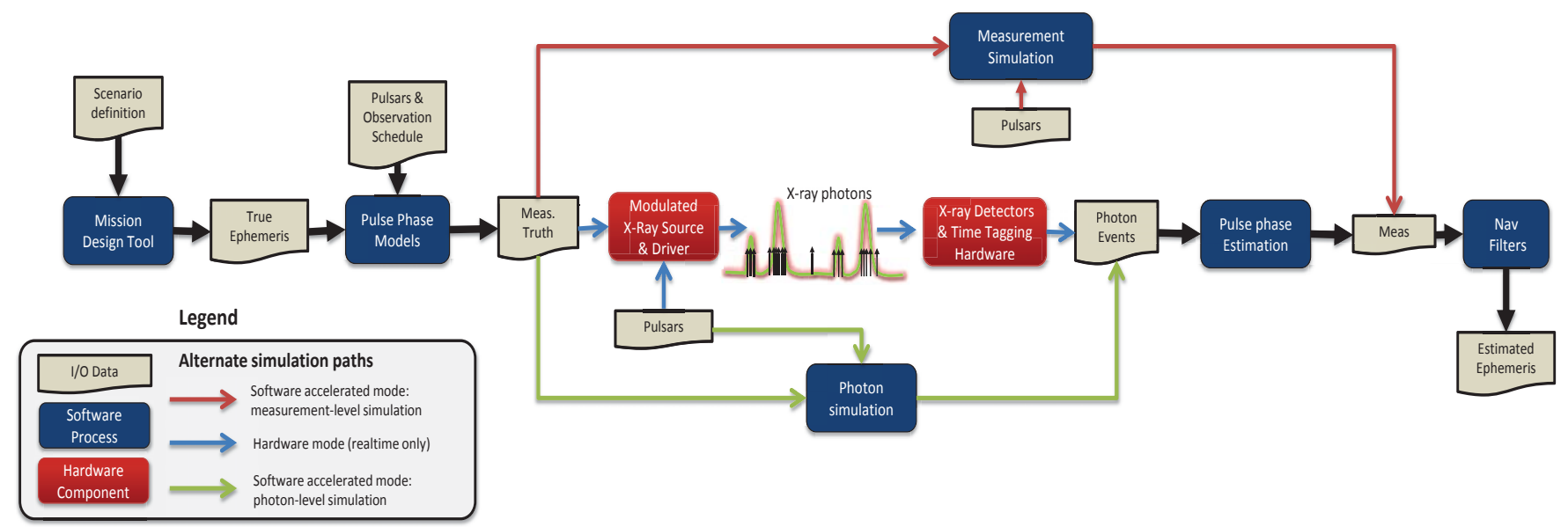

Figure 6: SEXTANT ground testbed architecture with three levels of simulation fidelity showing the flow from orbit simulation through truth measurement simulation, photon processing and navigation filtering algorithms.

and evaluate the phase model, as referenced to barycentric time, while accounting for parallax, Roemer delay, and Solar Shapiro delay.

Measurement generation-The simulation splits into three paths for measurement generation. At Level 0, measurements are generated by adding noise to the truth measurements consistent with the CRLB, plus a noise component used to capture additional noise sources. Individual photon events are not generated.

In Levels 1 and 2, photon events are created that can be input into the flight software directly. In Level 1, photon events are simulated using the algorithm described in Section 5. In Level 2, the photon events are generated in real-time by the MXS and timestamped by either a COTS, NICER ETU, or NICER flight detector package. Next, in both Levels 1 and 2, the events are delivered as a sequence of telemetry packets to the SEXTANT flight software. Once received, the software first pre-processes the events to filter out background events that fail a threshold test. Events that pass the preprocessor are buffered on a per-pulsar basis. When sufficient observation time is accumulated on a particular pulsar, the buffer is batch processed as described in Section 5. The output is a pulse phase and Doppler measurement suitable for processing by the navigation filter.

Navigation filter-Finally, the pulse phase and Doppler measurements are used to correct the spacecraft state estimate in the navigation filter. The SEXTANT flight software and end-to-end simulation use the XNAV-enhanced GEONS Extended Kalman Filter for navigation [16, 29]. The ground testbed also supports the NASA open source Orbit Determination Toolbox (ODTBX) filter software [30]. In either package, high fidelity models of orbital dynamics are used in conjunction with the measurement model described in Section 5 to propagate and update the spacecraft state. The propagated state history is stored and fed back to the measurement generation block to provide the phase predictor $\tilde{\phi}$ needed there.

\section{GXLT HARDWARE-IN-THE-LOOP MODE}

In this section, we describe, in more detail, the Level 2 HWIL simulation mode and its hardware components, as shown in
Figure 7. A key enabling component for the GXLT HWIL capability is GSFC's unique Modulated X-Ray Source.

\section{Modulated X-ray source}

Most common active X-ray sources use high voltage to accelerate electrons, emitted from a hot filament, that impinge upon a metal target at high velocity. These electrons produce $\mathrm{X}$-rays as they decelerate on target impact. Such conventional electron impact sources are modulated by varying the filament temperature or the accelerating potential, and consequently, suffer a modulation timescale on the order of $1 \mathrm{~s}$. Emulating the MSP X-ray environment requires considerably faster switching times. GSFC has developed a new kind of electron impact source, the Modulated X-Ray Source (MXS) seen in Figure 9, which uses an optical or Ultraviolet (UV) Light Emitting Diode (LED) to liberate photoelectrons from a photocathode [31] that are then accelerated toward a target at high potential. Thus, modulating the LED output modulates the photoelectron flux, which produces the modulated Xrays. The MXS X-ray output modulation is limited only by the LED. Commercially available LEDs support switching timescales on the order of nanoseconds, which enable the MXS to meet our environmental emulation need.

To simulate the photon arrival process as seen by a detector on a moving spacecraft, the MXS input LED is driven with a current signal proportional to the count rate function $\lambda(\phi(t))$ of the pulsar of interest. The task is achieved by the MXS driver.

\section{Modulated X-ray source driver}

The MXS driver is composed of a control program that runs on a PC, the MXS driver digital board, and an LED driver circuit, as shown in block diagram form in Figure 11. The control program takes as input the pulsar definitions and pulsar phase dynamics file, and produces a real-time implementation of $\lambda(\phi(t))$ as a precision current signal of appropriate level to drive the input of the MXS. This signal is precisely aligned to its internal master simulation timer which produces the simulation of UTC, $t$.

The control program, written in Python [32], runs on a standard PC and interfaces to the MXS driver digital board using an Ethernet connection. At each simulation time tick, the control program feeds target phase commands to the digital 


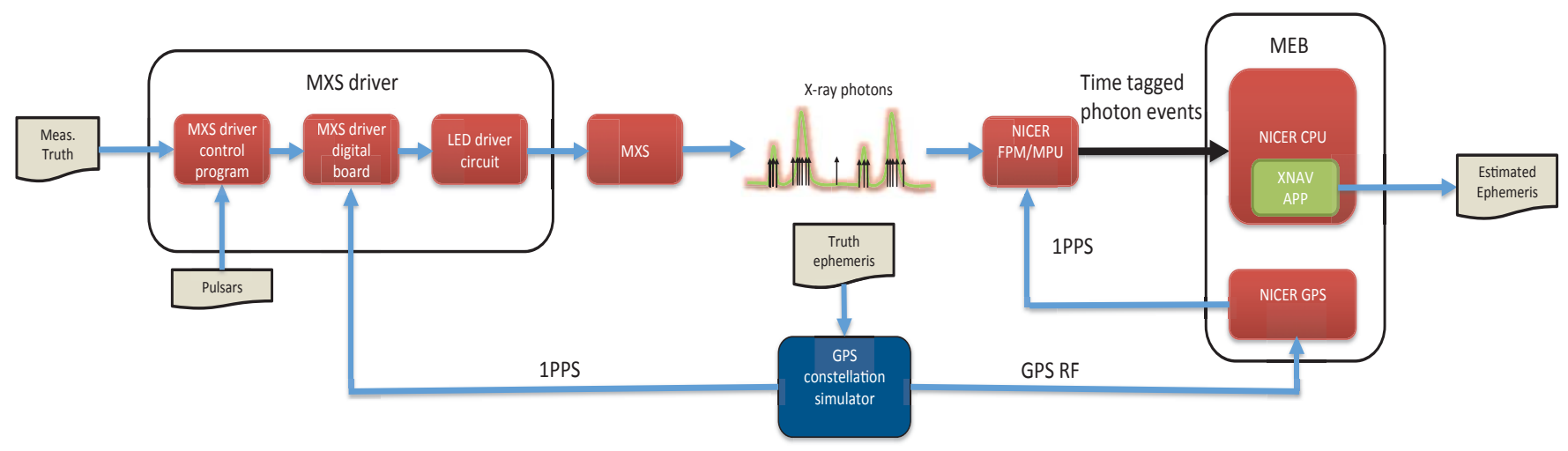

Figure 7: SEXTANT Level 2 simulation Hardware-in-the-Loop (HWIL) test configuration. The MXS is driven in real-time to produce X-rays with photon events following the simulation truth profile. The X-rays travel through a short gap and impact on the X-ray detector and are time-tagged using the FPM/MBU.

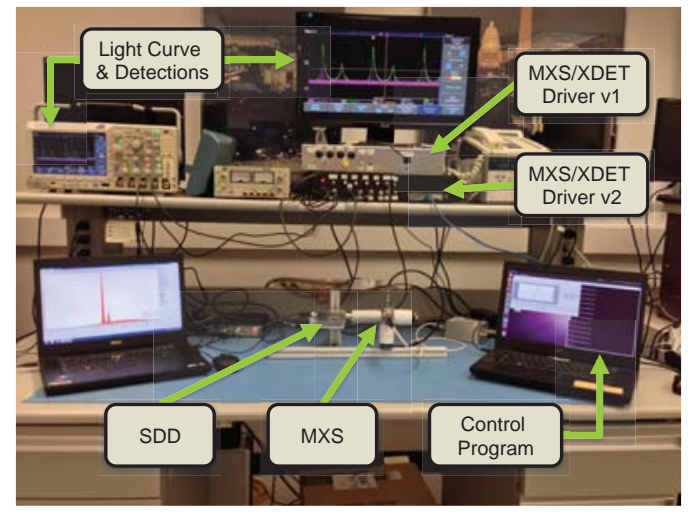

Figure 8: A photograph of the SEXTANT ground testbed.

board. The digital board is based on a Xilinx ML605 FieldProgrammable Gate Array (FPGA) card and consists of an embedded microprocessor that manages the communication with the hardware and custom logic blocks, all implemented in the FPGA. The custom logic implements a simulation timer, a Numerically Controlled Oscillator (NCO) for generating the pulse frequency, a look-up table that stores the pulse shape and relative count rates, and an interface to the Digital-to-Analog Converter (DAC) that produces the current signal which drives a custom LED driver analog circuit that ultimately modulates the LED.

The LED driver circuit, shown in Figure 10, converts the differential voltage output of the DAC to a precision current. It is composed of a four-stage amplifier circuit that provides necessary signal gain, independent controls to adjust the signal and background count rates, voltage-to-current conversion, and safety features to protect the MXS input diode.

\section{Testing with the pulsar simulator}

The output of the MXS is directed at an X-ray detector. A calibration procedure is then executed to ensure that the correct absolute count rates are obtained at the detector output by adjusting the channel from the MXS to the detector. Typically, this will be adjusted so the expected count rate from the entire XTI is seen in a single detector. Once absolutely calibrated, relative count rate changes upon switching from one source to another is faithfully reproduced by the MXS.

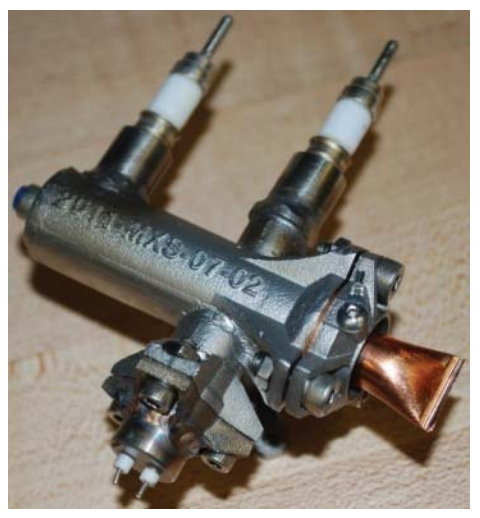

Figure 9: A photograph of the MXS.

For SEXTANT primary development, the MXS stimulates a NICER ETU detectors attached to the XTI timing chain, which includes a ETU flight computer that hosts the SEXTANT flight software. This configuration provides for a very high-fidelity on-orbit environment emulation.

In early development, prior to the arrival of the ETU detector package, a COTS SDD and a custom digital timestamping component, called the Time-Tagging X-Ray Detector (XDET), were used to time tag photon detections. The XDET consists of a control program running on a $\mathrm{PC}$ and a digital board board with an Analog-to-Digital Converter (ADC) attached. The output of the XDET is a list of photon time-of-arrivals stamped with simulated UTC time, which are stored in a file and then processed in the end-to-end simulation components as shown in Figure 6.

Figure 13(a) and 13(b) show Level 2 GXLT simulations of the Crab pulsar with high and low Signal-to-Noise Ratio (SNR), respectively. In these figures, three cycles of the MXS driving signal, emulating the rate function $\lambda(\phi(t))$ of the Crab pulsar, is shown on top of the resulting output of the X-ray detector for a low SNR (top (a)) and high SNR (bottom (b)) case. Each detected photon results in a impulse shown on the scope trace. The rate of events is proportional to the driving signal.

The MXS driver current signal is shown in blue along with the output of the detector, where each individual detected photons appear as green vertical lines. Detections occur at a rate proportional to the driving signal. Figure 14 shows the 


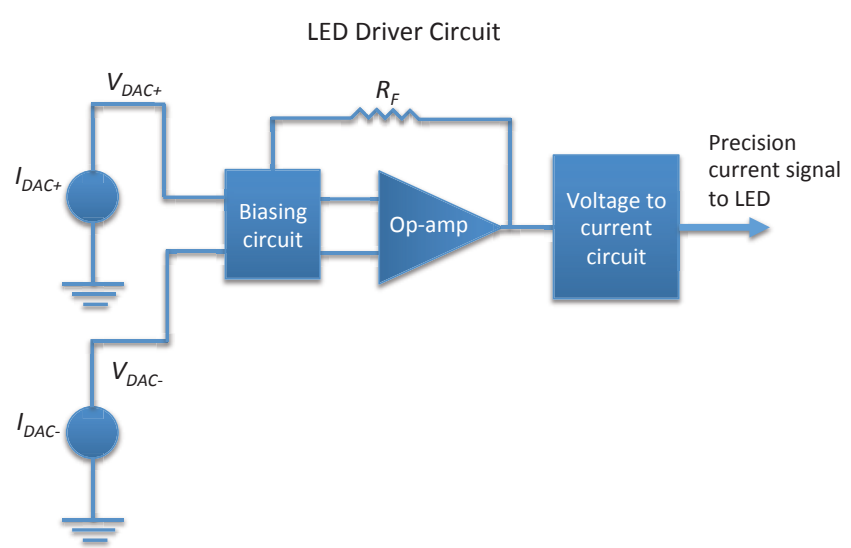

Figure 10: Block diagram of the LED driver circuit which is used to convert the differential voltage output of the DAC to a precision current that drives the input of the MXS.

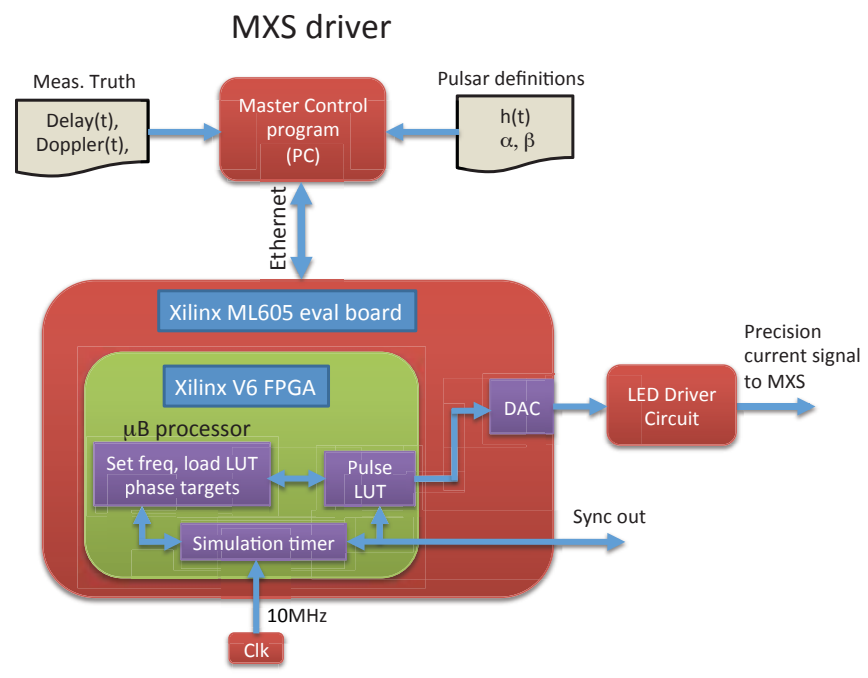

Figure 11: Block diagram of the MXS driver which consists of the Control Program, MXS driver digital board, and the LED driver circuit.

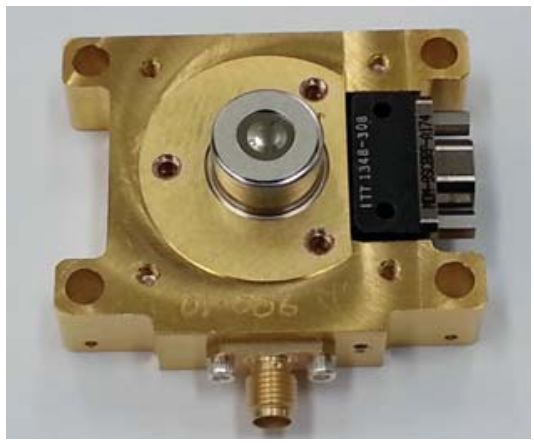

Figure 12: A photograph of a NICER X-ray detector.

Crab lightcurve clearly emerge as the result of folding the resulting events.

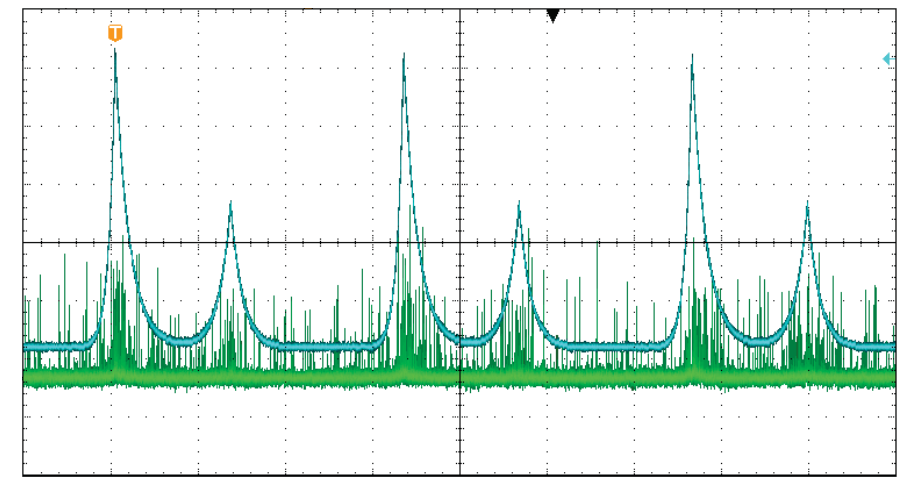

(a)

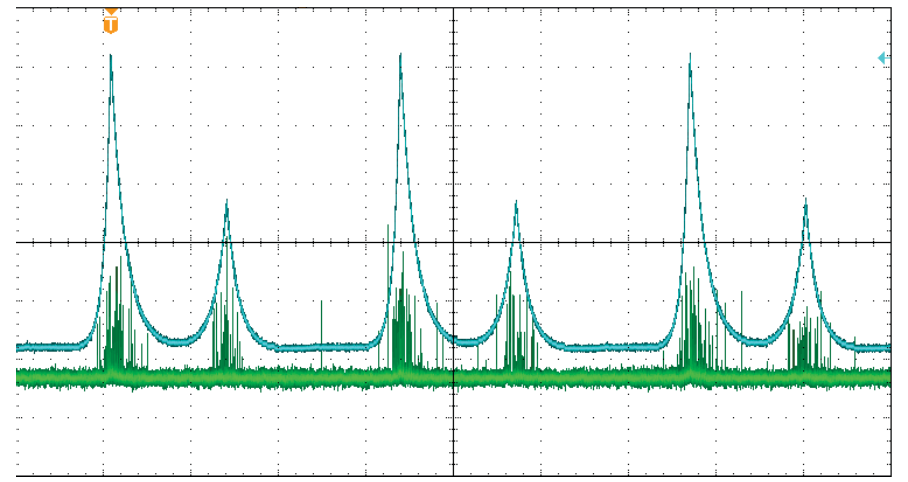

(b)

Figure 13: Simulated X-ray events from the GXLT Level 2 simulation captured on oscilloscope. (a) low SNR case (b) high SNR case.

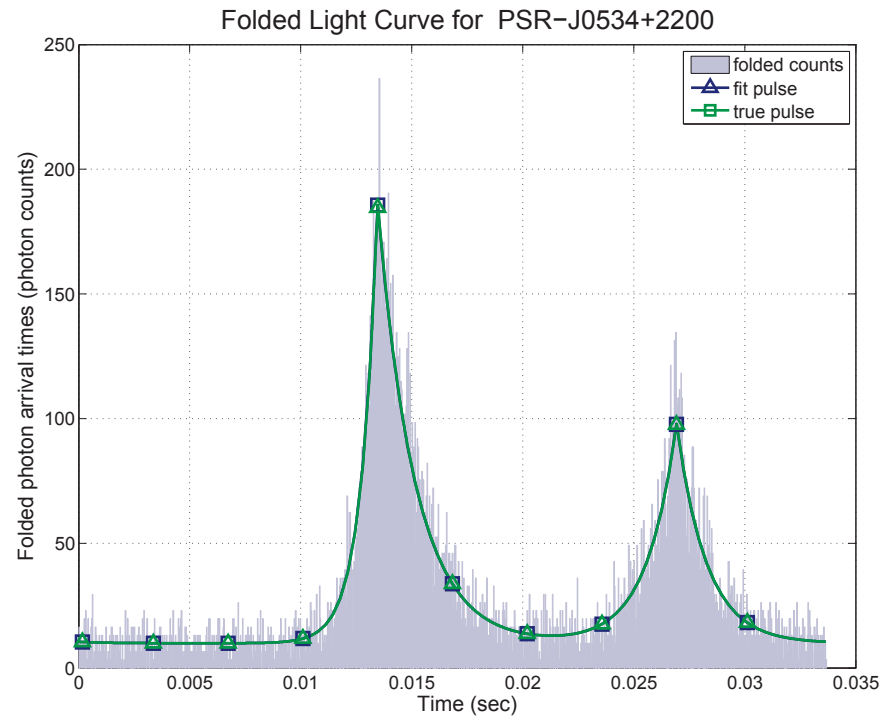

Figure 14: Simulated X-ray events from the GXLT Level 2 simulation captured to file and folded using the prediction.

\section{Preliminary Simulation Results}

In this section, we present simulation results using the SEXTANT's ground testbed end-to-end simulation capability, described in Section 6. The simulation described here models the SEXTANT primary experiment. 

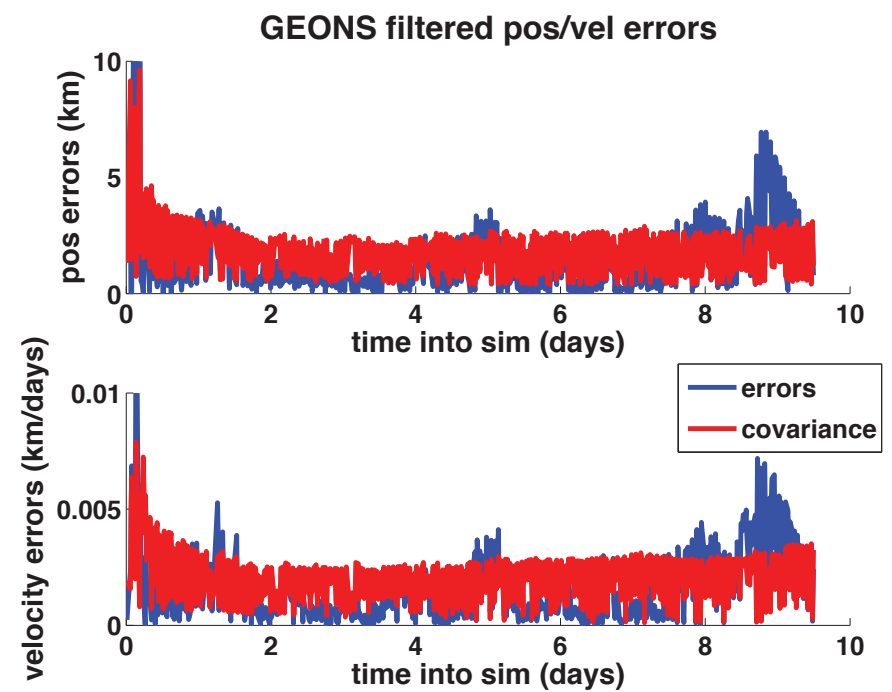

Figure 15: Results of the GXLT Level 1 simulation for a 9.5 day ISS simulation. This plot shows the RSS errors in position (top) and velocity (bottom) and their corresponding predicted 1-sigma variances.

Figure 15, shows results from the Level 1 end-to-end simulation for a 9.5 day simulation with cyclical measurements from four pulsars; B1937+21, J0437-4715, B1821-24 and J0218+4232. These results do not include models of the additional error sources (beyond Poisson noise) described in Section 5. This simulation is based on fixed observation periods of 1800 s each broken up over multiple contiguous time intervals if needed, taking into account Earth, Moon, and Sun occultation constraints. This simulation uses a geocenter reference observatory for the phase models $\phi_{0}$. The truth trajectory was generated in GMAT as described in Section 6. The GEONS initial state is seeded with GPS-like point solution with intentional biases $(1 \mathrm{~km}$ position and $1 \mathrm{~m} / \mathrm{s}$ velocity each axis) and noise $(1 \mathrm{~km}$ position and $1 \mathrm{~m} / \mathrm{s}$ velocity $1 \sigma$ each axis) added. The filter uses a JGM- $230 \times 30$ gravity model, and uses a fit to Harris-Priester drag model, but does not estimate the drag coefficient.

In this simulation (Figure 15), the navigation solution converges to a level well below the $10 \mathrm{~km}$ worst-direction objective.

\section{SUMMARY AND FUTURE WORK}

In this paper, we described, in detail, the algorithms and models at the core of the SEXTANT technology demonstration and the NASA GSFC GXLT that supports SEXTANT development, testing, and ground experiments. SEXTANT will demonstrate, for the first time, real-time on orbit XNAV with a stated primary objective of achieving absolute orbit determination to better than $10 \mathrm{~km}$ with 2 weeks of measurements in the highly dynamic LEO of the ISS. While the preliminary work contains some optimistic assumptions, the early simulation results are very encouraging and indicate that the primary objective can be met with margin.

A number of enhancements to the current baseline XNAV system implementation have been clearly identified and are in work. These enhancements have been discussed in the paper, and are summarized as: Implementation of a variable background model and integration of background estimation; Modeling of additional noise sources including reference clock errors, pulsar almanac model mismatch, intrinsic noise in the pulsars such as timing noise and glitches, uncertainty in the radio to X-ray phase offset, and astrometric errors; Enhancements to efficiently handle processing of the Crab Pulsar, which is an outlier in the Pulsar catalog, in that its flux is 5-6 orders of magnitude higher, and it requires timing ephemeris updates much more often than all other SEXTANT pulsars.

The NICER and SEXTANT completed a successful Critical Design Review (CDR) in September 2014 and continues to make excellent progress. Currently, the first version of the NICER flight software has been released for testing on a commercial development version of the flight processor, and includes the first release of the SEXTANT flight software. The second and final release of the SEXTANT XFSW is planned for early March of 2015, and will begin testing shortly there after.

Additionally, all photon events will be telemetered to the ground, archived, and made available to the public via NASA GSFC's HEASARC. SEXTANT will also use this data to support experiments using the GXLT.

\section{ACKNOWLEDGMENT}

The authors would like to thank Dr. Russell Carpenter for his valuable input and time.

This research has made use of data, software and/or web tools obtained from NASA's High Energy Astrophysics Science Archive Research Center (HEASARC), a service of Goddard Space Flight Center and the Smithsonian Astrophysical Observatory.

\section{REFERENCES}

[1] NASA, Systems Engineering Handbook, Revision 1. Washington, DC, USA: National Aeronautics and Space Administration, 2007, NASA/SP-2007-6105. [Online]. Available: http://go.usa.gov/Xdt3

[2] K. C. Gendreau, Z. Arzoumanian, T. Okajima, and the NICER Team, "The Neutron-star Interior Composition ExploreR (NICER): an Explorer mission of opportunity for soft X-ray timing spectroscopy," in Space Telescopes and Instrumentation: Ultraviolet to Gamma Ray, ser. Proc. SPIE, vol. 8443. International Society for Optics and Photonics, Sep 2012, doi:10.1117/12.926396.

[3] Z. Arzoumanian, K. C. Gendreau, C. L. Baker, T. Cazeau, P. Hestnes, J. W. Kellogg, S. J. Kenyon, R. P. Kozon, K.-C. Liu, S. S. Manthripragada, C. B. Markwardt, A. L. Mitchell, J. W. Mitchell, C. A. Monroe, T. Okajima, S. E. Pollard, D. F. Powers, B. J. Savadkin, L. B. Winternitz, P. T. Chen, M. R. Wright, R. Foster, G. Prigozhin, R. Remillard, and J. Doty, "The Neutron-star Interior Composition Explorer (NICER): Mission Definition," in Astronomical Telescopes and Instrumentation, ser. Proc. SPIE, vol. 9144. International Society for Optics and Photonics, 2014, pp. 914 420-914 420-9. [Online]. Available: http://dx.doi.org/10.1117/12.2056811

[4] S. I. Sheikh, "The use of variable celestial X-ray sources for spacecraft navigation," Ph.D. dissertation, University of Maryland, 2005. [Online]. Available: 
http://hdl.handle.net/1903/2856

[5] P. S. Ray, K. S. Wood, and B. F. Phlips, "Spacecraft navigation using X-ray pulsars," 2006 NRL Review, pp. 95-102, 2006.

[6] A. A. Emadzadeh and J. L. Speyer, Navigation in space by $X$-ray pulsars. Springer, 2011.

[7] G. S. Downs, "Interplanetary navigation using pulsating radio sources," NASA Technical Reports, N74-34150, pp. 1-12, 1974.

[8] T. J. Chester and S. A. Butman, "Navigation using Xray pulsars," Jet Propulsion Laboratory, Pasadena, CA, NASA Tech. Rep. 81N27129, 1981.

[9] K. S. Wood, "Navigation Studies Utilizing The NRL801 Experiment and the ARGOS Satellite," in Proc. SPIE, vol. 1940, 1993, pp. 105-116.

[10] W. Becker and J. Trümper, "Detection of pulsed Xrays from the binary millisecond pulsar J0437 - 4715," Nature, vol. 365, pp. 528-530, Oct. 1993.

[11] J. Sala, A. Urruela, X. Villares, R. Estalella, and J. M. Paredes, "Feasibility study for a spacecraft navigation system relying on pulsar timing information," European Space Agency Advanced Concepts Team ARIADNA Study, vol. 3, no. 4202, p. 23, 2004.

[12] X. P. Deng, G. Hobbs, X. P. You, M. T. Li, M. J. Keith, R. M. Shannon, W. Coles, R. N. Manchester, J. H. Zheng, X. Z. Yu, D. Gao, X. Wu, and D. Chen, "Interplanetary spacecraft navigation using pulsars," Advances in Space Research, vol. 52, pp. 1602-1621, Nov. 2013.

[13] P. Graven, J. Collins, S. Sheikh, J. Hanson, P. Ray, and K. Wood, "XNAV for deep space navigation," in 31st Annual AAS Guidance and Control Conference, 2008, pp. 08-054. [Online]. Available: http://microcosminc. com/analysis/AAS\%2008-054\%20XNAV.pdf

[14] H. Wolter, "Spiegelsysteme streifenden Einfalls als abbildende Optiken für Röntgenstrahlen," Annalen der Physik, vol. 445, pp. 94-114, 1952.

[15] J. W. Mitchell, M. A. Hassouneh, L. M. Winternitz, J. E. Valdez, P. S. Ray, Z. Arzoumanian, and K. C. Gendreau, "Station Explorer for X-ray Timing and Navigation Technology Architecture Overview," in Proceedings of the 27th International Technical Meeting of the Satellite Division of the Institute of Navigation (ION GNSS+). Institute of Navigation, 2014.

[16] A. Long, Goddard Enhanced Onboard Navigation System (GEONS) Mathematical Specifications, a.i. solutions Inc., November 2012, FDSS-23-0035, Version 2, Release 2.17.

[17] NASA Goddard Space Flight Center, "High Energy Astrophysics Science Archive Research Center," http: //heasarc.gsfc.nasa.gov/, Nov. 1990, retrieved Oct 10, 2014.

[18] G. B. Hobbs, R. T. Edwards, and R. N. Manchester, "TEMPO2, a new pulsar-timing package - i. an overview," Monthly Notices of the Royal Astronomical Society, vol. 369, pp. 655-672, Jun. 2006. [Online]. Available: http://adsabs.harvard.edu/abs/2006MNRAS. $369 . .655 \mathrm{H}$

[19] R. T. Edwards, G. B. Hobbs, and R. N. Manchester, "TEMPO2, a new pulsar timing package - II. the timing model and precision estimates," Monthly Notices of the Royal Astronomical Society, vol. 372, pp. 1549-1574, Nov. 2006. [Online]. Available: http://adsabs.harvard.edu/abs/2006MNRAS.372.1549E

[20] L. Leemis, "Estimating and simulating nonhomogenous Poisson processes," William and Mary Mathematics Department, May 2003, retrieved Sep 5 2012. [Online]. Available: http://www.math.wm.edu/ leemis/icrsa03. pdf

[21] J. F. C. Kingman, Poisson processes, ser. Oxford Studies in Probability. New York: The Clarendon Press Oxford University Press, 1993, vol. 3, oxford Science Publications.

[22] J. R. Carpenter, "Xnav photon processing,” Apr. 2014, personal communication.

[23] I. Bar-David, "Communication under the poisson regime," Information Theory, IEEE Transactions on, vol. 15, no. 1, pp. 31-37, Jan 1969.

[24] A. R. Golshan and S. I. Sheikh, "On pulse phase estimation and tracking of variable celestial X-ray sources," in Proceedings of the 63rd Annual Meeting of The Institute of Navigation. Cambridge, MA: Institute of Navigation, April 2007, pp. 413-422.

[25] G. Casella and R. Berger, Statistical Inference. Duxbury Resource Center, June 2001.

[26] X. P. Deng, W. Coles, G. Hobbs, M. J. Keith, R. N. Manchester, R. M. Shannon, and J. H. Zheng, "Optimal interpolation and prediction in pulsar timing," MNRAS, vol. 424, pp. 244-251, Jul. 2012.

[27] L. M. B. Winternitz, M. A. Hassouneh, J. A. Gaebler, J. W. Mitchell, F. Gavriil, Z. Arzoumanian, and K. C. Gendreau, "An X-ray Navigation Ground Testbed," in 27th Space Simulation Conference. IEST, November 2012.

[28] NASA JSC, “Orbital Elements from Johnson Space Center's Flight Design and Dynamics Division," 2014, accessed: 2014-02-06. [Online]. Available: http:// spaceflight.nasa.gov/realdata/sightings/SSapplications/ Post/JavaSSOP/orbit/ISS/SVPOST.html

[29] A. Long, Goddard Enhanced Onboard Navigation System (GEONS) System Description and User's Guide, a.i. solutions Inc., November 2012, FDSS-23-0026, Version 2, Release 2.17.

[30] K. M. Getzandanner, "Orbit Determination Toolbox (ODTBX)," NASA Goddard Space Flight Center Navigation and Mission Design Branch, retrieved Sep 12, 2012. [Online]. Available: http://odtbx.sf.net/

[31] K. Gendreau, Z. Arzoumanian, P. Deines-Jones, and R. Koenecke, "A modulated X-ray source for in-flight calibration of high-energy astrophysics instrumentation," 2011, unpublished.

[32] "The Python language reference," retrieved Sep 23, 2012. [Online]. Available: http://docs.python.org/ reference/

\section{BIOGRAPHY}

Dr. Luke M.B. Winternitz is an engineer at NASA GSFC in the Components and Hardware Systems branch. He is the SEXTANT Systems Architect and the technical lead of the space-GPS receiver research and development group. He received his Ph.D. (2010) in Electrical Engineering from the University of Maryland, College Park.

Dr. Munther A. Hassouneh is an engineer at NASA GSFC in the Components and Hardware Systems branch. He is the SEXTANT ground testbed lead and is a member of the space-GPS receiver research and development group. He received his Ph.D. (2003) in Electrical Engineering from the University of Maryland, College Park. 
Dr. Jason W. Mitchell is an engineer at NASA GSFC in the Navigation and Mission Design branch, and is the project manager for the Station Explorer for X-ray Timing and Navigation Technology (SEXTANT). He received his Ph.D. (2000) in Aerospace Engineering from the University of Cincinnati.

Ms. Jennifer E. Valdez is an engineer at NASA GSFC in the Components and Hardware Systems branch. She is the SEXTANT lead for the XNAV end-to-end simulator and is a member of the space-GPS receiver research and development group.

Mr. Wayne H. Yu is an engineer at NASA GSFC in the Navigation and Mission Design Branch. He performs navigation and flight dynamics analysis for the XNAV end-to-end simulator, focusing on visibility and scheduling modeling.

Dr. Sean R. Semper is an engineer at NASA GSFC in the Components and Hardware Systems branch and he is the SEXTANT ground system lead. He received his Ph.D. (2011) in Aerospace Engineering from the University at Buffalo, SUNY.

Dr. Paul S. Ray is an astrophysicist at the Naval Research Laboratory. He is a NICER science team member specializing in high precision timing observations of pulsars. He received his Ph.D. (1995) in Physics from the California Institute of Technology.

Dr. Kent S. Wood is an astrophysicist at the Naval Research Laboratory. He is a NICER science team member specializing in pulsar observations and observations involving other celestial source classes, as well as calibrations and background modeling. He received his Ph.D. (1973) in Physics from the Massachusetts Institute of Technology.

Dr. Zaven Arzoumanian is a Senior Research Scientist with the Universities Space Research Association (USRA), working within the Center for Research and Exploration in Space Science and Technology (CRESST) at NASA's Goddard Space Flight Center. He currently serves as Deputy Principal Investigator and Science Lead for NICER.

Dr. Keith C. Gendreau is currently the principal investigator of the X-ray Advanced Concepts Test Bed sounding rocket, the SEXTANT demonstration, and the NICER mission. He received his Ph.D. (1995) in Astrophysics from the Massachusetts Institute of Technology, and was the NASA scientist on the joint NASA-DARPA $X$-ray pulsar source-based navigation and timing study. 